\title{
Double transition-metal MXenes: Atomistic design of two-dimensional carbides and nitrides - ADDENDUM
}

\section{Weichen Hong*, Brian C. Wyatt*, Srinivasa Kartik Nemani*, and Babak Anasori}

DOI: https://doi.org/10.1557/mrs.2020.251 Published online by Cambridge University Press: 09 October 2020

\section{Reference}

1. W. Hong, B. Wyatt, S. Nemani, \& B. Anasori, Double transition-metal MXenes: Atomistic design of two-dimensional carbides and nitrides. MRS Bulletin, 45(10), 850-861. (2020) 